\title{
HORTICULTURA PERIURBANA, ANÁLISIS DE LA FERTILIDAD DE LOS SUELOS EN INVERNADEROS
}

\section{PERI-URBAN HORTICULTURE, ANALYSIS OF SOIL FERTILITY IN GREENHOUSES}

\author{
Marisol Virginia Cuellas ${ }^{1 *}$ \\ ${ }^{1}$ Agencia de Extensión La Plata, Estación Experimental del Área Metropolitana de Buenos Aires, \\ Instituto Nacional de Tecnología Agropecuaria (INTA), Ruta 36 Km 44,5 (1900 La Plata), Argentina. \\ *Autor para correspondencia E-mail: cuellas.marisol@inta.gob.ar
}

\section{RESUMEN}

La horticultura periurbana del Gran La Plata, Argentina, es muy importante, ya que provee de hortalizas frescas a 13 millones de habitantes, y si bien tiene la ventaja de cercanía a la ciudad, debe afrontar problemas vinculados a procesos tecnológicos, siendo un sistema productivo frágil y con elevado riesgo de impacto ambiental. Los suelos en los cuales se desarrolla la producción bajo cubierta se los clasifica como Vertic Argiudol y Typic Hapludert, y en ellos se cultivan un gran número de especies, principalmente tomate (Solanum lycopersicum), pimiento (Capsicum annuum. L.) y lechuga (Lactuca sativa L.), se los maneja sin realizar análisis de suelo, utilizando una gran cantidad de insumos (fertilizantes, enmiendas, etc.) con consecuencias ambientales (contaminaciones, degradaciones) y productivas (deficiencias inducidas, costos elevados). El presente trabajo tuvo como objetivo hacer un diagnóstico de la fertilidad química de los suelos, utilizando análisis de rutina, estableciendo su vinculación con el desarrollo de los cultivos. Se observó, en la mayoría de los casos, salinización (promedio $4 \mathrm{dS} \mathrm{m}^{-1}$ ), alcalinización, sodificación (PSI 18\%), pérdida de materia orgánica (promedio 3,4\%) e hiperfertilización (concentraciones de P hasta $535 \mathrm{mg} \mathrm{kg}^{-1}$ ) con efectos sobre los cultivos. Por consiguiente, si en la región el manejo de los suelos continúa efectuándose sin un correcto ajuste a los tipos de suelos y a las necesidades de los cultivos, la producción de hortalizas estará seriamente afectada, y los procesos de contaminación y degradación serán cada vez más intensos.

Palabras clave: deficiencias inducidas, salinización, sodificación, hiperfertilización, hortalizas.

\section{ABSTRACT}

The peri-urban horticulture of Gran La Plata produces fresh vegetables for 13 million inhabitants. Although production is located close to the city, it presents problems associated with technological processes, and represents a fragile production system with a high environmental impact risk. Soils used for the production of horticultural crops under cover are classified as Vertic Argiudol and Typic Hapludert. A large number of species, mainly tomatoes (Solanum lycopersicum), peppers (Capsicum annuum. L.) and lettuce (Lactuca sativa L.), are grown without conducting soil tests and applying high amounts of fertilizers and/or amendments, which results in environmental (pollution, degradation) and productive (induced deficits, high costs) consequences. This study aimed at making a diagnosis of the chemical soil fertility, using routine soils tests, and determining its relationship with crop development. In most cases, salinization (average $4 \mathrm{dS} \mathrm{m}^{-1}$ ), alkalization and sodification 
(PSI 18\%), as well as loss of organic matter (average 3.4\%) and hyperfertilization (P concentrations up to $535 \mathrm{mg} \mathrm{kg}^{-1}$ ) affected crops. Therefore, if soil management continues without proper adjustment to soil types and crop requirements, not only vegetable production will be seriously affected, but contamination and degradation will also increase.

Key words: induced deficiency, salinization, sodification, hyperfertilization, vegetables.

\section{INTRODUCCIÓN}

La agricultura periurbana se concentra en los alrededores de las grandes ciudades, y conforma los denominados "cinturones verdes", en donde se encuentra un entramado de explotaciones primario intensivas, quemanejanungran volumen de producción (Barsky, 2005). La horticultura periurbana del área metropolitana de Buenos Aires, el Cinturón Hortícola Metropolitano (CHM) es muy importante, debido a que satisface gran parte de la demanda de hortalizas frescas de 13 millones de habitantes. Si bien las producciones hortícolas del CHM presentan como ventaja competitiva la proximidad a la ciudad, deben afrontan diferentes problemáticas, vinculadas a esta proximidad y a procesos tecnológicos como la falta de conocimiento y concientización de los procesos de contaminación de los recursos (agua, suelo y aire), el bajo uso de tecnologías apropiadas, el deficiente manejo de sustratos y suelos, entre otras (Mitidieri, 2014). En general, se caracterizan por un uso desmedido de insumos (fertilizantes, plaguicidas, desinfectantes, y abonos) que deterioran los recursos naturales (Polack, 2013), siendo la consecuencia principal un sistema productivo frágil y con elevado riesgo de impacto ambiental.

Dentro del CHM se destaca la región del Gran La Plata, por ser una de las zonas de mayor importancia a nivel nacional en la producción hortícola en invernadero. Se determinó para el año 2011 una superficie bajo cubierta de 2.251 ha (López Camelo, 2011). Se cultivan más de 15 especies, destacándose los cultivos de tomate (Solanum lycopersicum), pimiento (Capsicum annuum. $L$ ) y lechuga (Lactuca sativa $L$ ). Los suelos donde se desarrolla esta actividad se clasifican como Typic Hapludert y Vertic Argiudol (Hurtado et al., 2006). Presentan naturalmente elevada fertilidad química, alto nivel de materia orgánica $(4,5-5 \%)$ y están libres de sales y sodio (CE $<1$ dS $\mathrm{m}^{-1} \mathrm{y}$ RAS $<1$ ), su pH es ligeramente ácido ( $\left.\mathrm{pH} 5,5\right)$. Como única limitante química se destaca el bajo contenido de fósforo $\left(<10 \mathrm{mg} \mathrm{kg}^{-1}\right)$. No obstante, la propiedad que condiciona el manejo de estos suelos es la permeabilidad moderadamente baja a baja y la elevada plasticidad, debido a que poseen un elevado contenido de arcillas (entre $32-40 \%$ en superficie) que se incrementan en profundidad
(50-60\%), prevaleciendo la illita y en segundo término la montmorillonita (Hurtado et al., 2006). Estas limitantes naturales, sumadas al riego con agua de baja calidad (bicarbonatada sódica) (Alconada y Zembo, 2000) y al manejo productivo que se implementa (exceso de fertilización con enmiendas orgánicas e inorgánicas), pueden provocar degradaciones en el suelo de tipo físicas, físico-químicas, y biológicas, afectando también el crecimiento de los cultivos (Poncetta et al., 2006).

La acumulación de sales en el suelo es el factor principal que limita la producción en los cultivos bajo cubierta, siendo el mayor responsable la aplicación en exceso de fertilizantes inorgánicos y en segundo lugar la evapotranspiración que favorece la acumulación de sales en superficie (Zhang et al., 2006). En la región de estudio, Cuellas (2015) ha detectado valores de hasta 9,8 $\mathrm{dS} \mathrm{m}^{-1}$ de conductividad eléctrica. En condiciones de salinidad, se produce un desbalance nutricional, por efecto de diferentes factores como la disponibilidad de los nutrientes y la absorción competitiva. Cada cultivo tiene una respuesta diferente, que está en función de su umbral de tolerancia y del estado fenológico en el que se encuentra (Porta et al., 2003). Así se destaca, que a partir de valores de CE de 1,25 dS $\mathrm{m}^{-1}$ se ve afectado el rendimiento de la lechuga, y con concentraciones más elevadas $\left(1,5\right.$ y $\left.2 \mathrm{dS} \mathrm{m}^{-1}\right)$ se verán afectados los rendimientos de pimiento y tomate, respectivamente (Nuez et al., 2003, Cadahia, 2005).

Si bien los cultivos intensivos, principalmente los de fruto, presentan altos requerimientos nutricionales, a fin de compensar la alta producción de biomasa y obtener alta calidad de fruta (Cadahia, 2005), es imprescindible que la nutrición sea adecuada a la demanda. La fertilización influye en el balance nutricional de las plantas, por lo tanto cuando la misma no se adecúa a las necesidades nutricionales y a las condiciones edáficas, genera desbalance nutricional, siendo frecuente la ocurrencia de deficiencias inducidas y mayor incidencia de plagas y enfermedades en los cultivos (Altieri and Nicholls, 2003).

Los desórdenes fisiológicos, tales como el déficit de $\mathrm{Ca}^{2+}$, denominados "blossom end $\operatorname{rot}^{2}$ (necrosis apical del fruto) en tomate y pimiento, o 
tip burn (quemado de las puntas de las hojas) en lechuga, pueden tener distintos orígenes, como excesos iónicos de $\mathrm{K}^{+}, \mathrm{Na}^{+}, \mathrm{Mg}^{+2}, \mathrm{y} \mathrm{NH}_{4}^{+}, \mathrm{Mn}^{+2}$, estrés por agua, alta salinidad y alta humedad relativa, más que corresponderse específicamente a un bajo contenido de $\mathrm{Ca}^{+2}$ edáfico, por lo tanto el exceso de un elemento o las interacciones nutritivas en muchos casos pueden resultar perjudiciales (Saure, 2001; De Pascale et al., 2003; Ho and White, 2005; del Amor and Marcelis, 2006).

Para contrarrestar los efectos negativos en el suelo y en los cultivos, sin realizar un análisis de la situación, en la región de estudio se aumentan las dosis de fertilización, provocando hiperfertilización en los suelos (Alconada et al., 2011); así, se han encontrado valores de hasta $300 \mathrm{mg} \mathrm{kg}^{-1}$ de P (Guiffré et al., 2004). Situaciones similares se han reportado en otros sitios del mundo (Johnstone et al., 2005). Se destaca también que en la región se ha detectado contaminación de las aguas subterráneas con nitratos (Auge et al., 2004).

En los diagnósticos realizados por el Instituto Nacional de Tecnología Agropecuaria (INTA 2011a; 2011b), en el Gran La Plata, destacan como principales problemas productivos, la falta de incorporación de tecnologías de bajo impacto ambiental, la contaminación por el uso excesivo y sin control de agroquímicos, la contaminación microbiológica por la utilización de estiércoles, la acumulación de residuos y la degradación de los suelos vinculada al uso intensivo de los mismos. Se debe entonces iniciar un proceso de transición hacia el rediseño del sistema productivo, por lo tanto el manejo adecuado de los suelos adquiere gran relevancia para la sustentabilidad de los sistemas.

El presente trabajo tuvo como objetivo hacer un análisis general de las condiciones actuales en los que se encuentran los suelos del Gran La Plata en los que se realiza horticultura bajo cubierta, evaluando y estudiando las características químicas y físicoquímicas de los mismos y determinando el posible efecto en los cultivos.

\section{MATERIALES Y MÉTODOS}

Selección de sitios. La región de estudio fue el Gran La Plata (latitud $34^{\circ} 50^{\prime}$ y $35^{\circ} 30^{\prime}$ S y longitud $57^{\circ} 45^{\prime}$ y 5820' O), Argentina. Se realizó un muestreo teórico no probabilístico, donde la selección de casos no buscó conseguir la representatividad estadística del universo de actores de la región, sino que los sitios fueron elegidos por su relevancia en las características de la investigación. Así se seleccionaron 36 sitios de producción hortícola, los que cumplieron con tener más de tres años bajo cubierta, con similares características productivas, en donde se observaron en el suelo síntomas de salinización como eflorescencias blanquecinas y además el productor manifestó tener problemas vinculados al manejo de los suelos.

Descripción de los sitios de estudio. Todas las quintas, por su sistema de producción se caracterizaron como "familiar o familiar capitalizado" (INTA 2011a; 2011b), con superficies prediales que variaron entre 1 y 2 ha. El esquema productivo de cada establecimiento era intensivo y diversificado, siendo las principales especies cultivadas tomate, pimiento y lechuga, por lo que la interpretación de resultados se realizó en base a estos tres cultivos.

El sistema productivo implementado es únicamente bajo coberturas plásticas, en invernaderos "tipo capilla" (a dos aguas) de madera, característicos de la zona. Los manejos habituales consideran la aplicación de yeso en dosis variables, con una secuencia de labores descrita a continuación:

- Cincelado o subsolado: En general se realiza con el objetivo de romper la capa superior o bien de desarmar los lomos del cultivo anterior.

- Arado rotativa: Con el objeto de mezclar el yeso, como así también para adecuar el tamaño del terrón, preparar la cama de plantación para recibir el plantín.

- Construcción de lomos. En promedio los lomos tienen entre 0,60-0,80 m de ancho y el largo depende del tamaño del invernadero en general se encuentran entre 50-100 m.

- Desinfección del suelo: Desinfección química con Bromuro de metilo o 1,3 Dicloropropeno + clopicrina (dosis de ambos productos $50 \mathrm{gr} \mathrm{m}^{-2}$ ).

- Aplicación de enmienda orgánica: Cama de pollo o gallinaza, en dosis de $40 \mathrm{tha}^{-1}$, una o dos veces al año. Las características de estos productos son variables.

- Finalizada la etapa de preparación del suelo, se implanta el cultivo, con su correspondiente sistema de riego y se determina la fertilización. Generalmente el sistema de riego es por goteo (20 cm entre goteros, con dos mangueras de riego por lomo) y la fertilización se realiza por fertirrigación. Si bien esta práctica está muy difundida, la realidad es que en muchos casos se aplica una "receta de fertilización" sin considerar las características físico-químicas del suelo de cada quinta y sin adecuarla a la demanda del cultivo (Szczesny et al., 2014). Como ejemplo de fertilización se puede mencionar: Nitrato de Calcio $\left(\left(\mathrm{NO}_{3}\right)_{2} \mathrm{Ca}\right)$ y Nitrato de Potasio $\left(\left(\mathrm{NO}_{3}\right) \mathrm{K}\right)$ en dosis de 360 y $540 \mathrm{~kg} \mathrm{ha}^{-1}$, respectivamente. En muchos sitios también se aplica ácido fosfórico en dosis $240 \mathrm{~L} \mathrm{ha}^{-1}$. 
Muestreo y análisis de suelo. Se tomaron muestras (durante los años 2013-2015) de suelo en 36 sitios, a una profundidad de muestreo de 0-15 cm, previo a la implantación del cultivo; todas las muestras fueron compuestas (formadas por cuatro sub-muestras). Se analizaron las siguientes variables por estándares de evaluación (Page et al., 1982): pH en pasta, conductividad eléctrica (CE) en el extracto de suelo a saturación, capacidad de intercambio catiónico (CIC) por extracción con acetato de amonio $1 \mathrm{~N} \mathrm{pH} \mathrm{7,}$ cationes intercambiables, por extracción con acetato de amonio $1 \mathrm{~N} \mathrm{pH} 7$, y posterior evaluación con la metodología indicada para cada catión: fotometría en llama para sodio $\left(\mathrm{Na}^{+}\right)$y potasio $\left(\mathrm{K}^{+}\right)$y colorimetría para calcio $\left(\mathrm{Ca}^{+2}\right)$ y magnesio $\left(\mathrm{Mg}^{+2}\right)$; además se midió fósforo $(\mathrm{P})$ asimilable por Bray Kurtz 1 y Materia orgánica (MO) por Walkley y Black.

Análisis de datos. Cada variable se analizó estableciendo promedios, máximos y mínimos. Se realizaron correlaciones (coeficiente de Pearson) entre las mismas.

\section{RESULTADOS Y DISCUSIÓN}

Salinidad. En la Fig. 1 se representa el porcentaje de sitios evaluados que se encuentran en cada rango de salinidad (CE), establecido de acuerdo a la tolerancia de cada cultivo (lechuga, pimiento, tomate). Se observa que un porcentaje muy bajo $(16,7 \%)$ de todos los sitios, presentaron niveles de salinidad de hasta 1,25 dS m $\mathrm{d}^{-1}$ (con mínimos de $\left.0,7 \mathrm{dS} \mathrm{m}^{-1}\right)$. Si se considera como base el cultivo de lechuga, que es el de menor tolerancia salina, con estos niveles los cultivos considerados (lechuga, tomate y pimiento) se desarrollarán con normalidad. No obstante, en la mayoría de los sitios $(83,3 \%)$ el rendimiento de la lechuga se verá afectado, y la intensidad con que se afecte dependerá de la concentración salina (Cadahia, 2005). Así Ünlükara et al. (2010) determinaron que a partir de $1,1 \mathrm{dS} \mathrm{m}^{-1}$, por cada unidad de CE que se aumente, la lechuga reducirá su rendimiento en aproximadamente un $9-10 \%$.

Respecto a los cultivos de pimiento y tomate, si bien tienen mayor tolerancia salina que el cultivo de lechuga (umbrales de 1,5 y 2 dS $\mathrm{m}^{-1}$, respectivamente) (Nuez et al., 2003), se encontraron igualmente comprometidos en su desarrollo y producción, debido a que la mayoría de los sitios $(72 \%)$ presentaron salinidades $\geq 2$ $\mathrm{dS} \mathrm{m}^{-1}$, y dentro de este porcentaje, en un $36 \%$ la salinidad fue mayor a los $4 \mathrm{dS} \mathrm{m}^{-1}$, con extremos que alcanzaron los 10,6 dS $\mathrm{m}^{-1}$ (Fig. 1). Se destaca que el tomate reduce un $6 \%$ su rendimiento por cada unidad de CE que se incrementa a partir de los 2,5 dS $\mathrm{m}^{-1}$ (Maggio et al., 2007) y el pimiento $10,9 \%$ a partir de los 1,2 $\mathrm{dS} \mathrm{m}^{-1}$ (Kurunc et al., 2014).

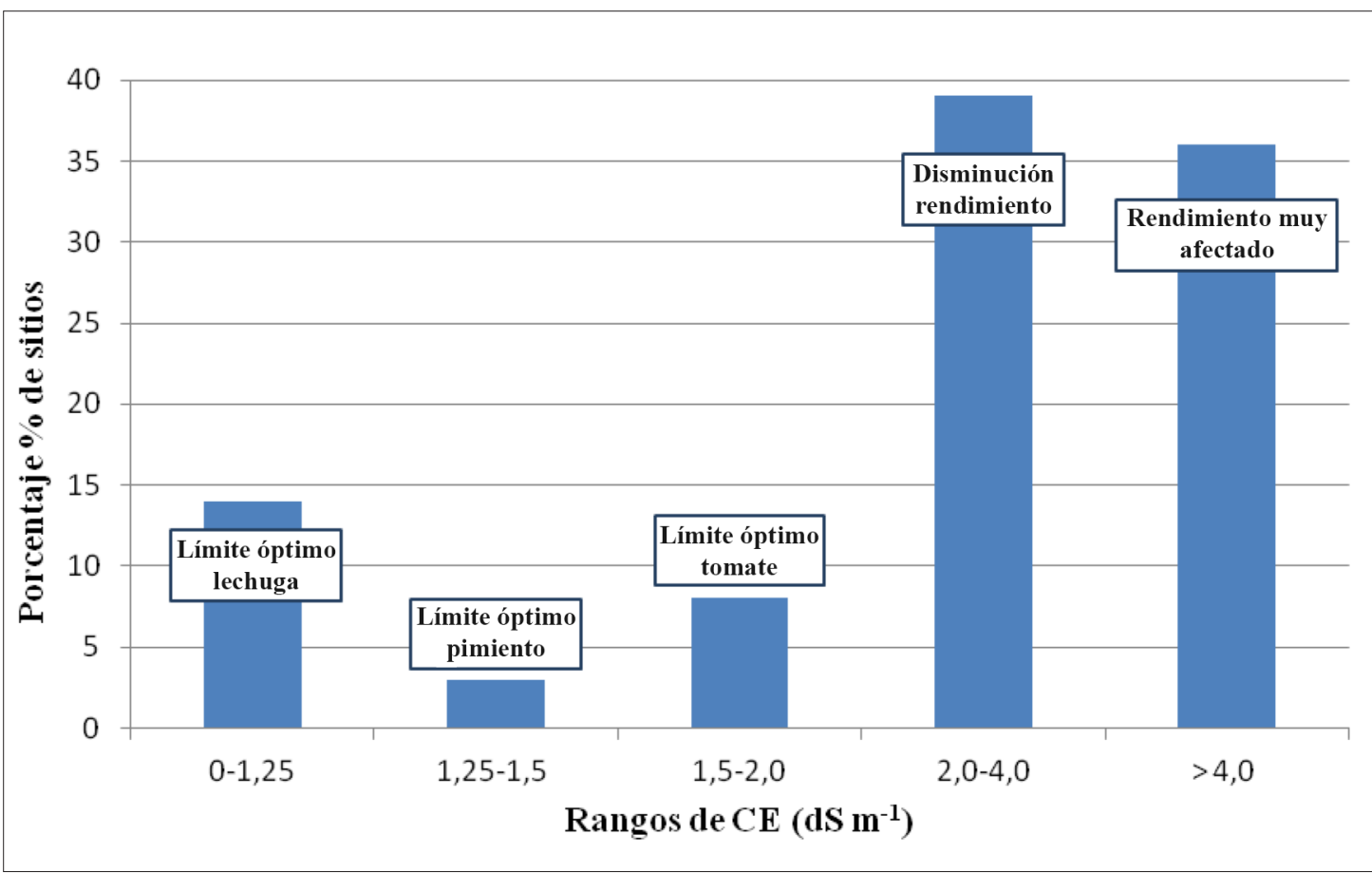

Fig. 1. Distribución porcentual (\%) de los sitios según el rango de CE (dS $\left.\mathrm{m}^{-1}\right)$

Fig. 1. Percentage distribution (\%) of the sites according to the EC range ( $\left.\mathrm{dS} \mathrm{m}^{-1}\right)$ 
$\mathrm{Si}$ bien los valores encontrados fueron en algunos casos menores a los reportados en la

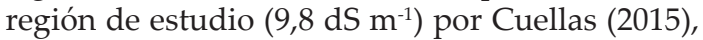
con las concentraciones salinas que se manifiestan en los suelos, los cultivos de mayor importancia en la región tendrán seriamente afectado su rendimiento, debido a que con estos niveles de salinidad las plantas sufren un desbalance nutricional, un déficit hídrico y una toxicidad por iones específicos (Porta et al., 2003). Al respecto, Rubio et al. (2009) determinaron que la salinidad reduce el rendimiento y la proporción de frutos comerciales, principalmente porque se aumenta el número de frutos con blossom end rot (BER). En los casos de este estudio, los productores incorporan cada vez más insumos externos (fertilizantes, cama de pollo, etc.) para atenuar la disminución de los rendimientos, sin encontrar una respuesta certera, aumentando así los costos y la degradación de los suelos (Poncetta et al., 2006; Alconada et al., 2011). Se destaca que las enmiendas orgánicas (camas de pollo) de la zona de estudio alcanzan valores elevados de salinidad, con mínimos de 3 y máximos de 8 dS $\mathrm{m}^{-1}$ (datos no mostrados).

Reacción del suelo (pH). En la Tabla 1 se presentan los valores de $\mathrm{pH}$ para todos los sitios evaluados. Se observa una alcalinidad generalizada, con valores promedios de 7,5 y extremos de 8,6. Nuez et al. (2003) establecieron para los cultivos hortícolas evaluados, un crecimiento óptimo en un rango de $\mathrm{pH}$ entre 5,5-6,5. En el presente estudio, excepto por un sitio, todos superaron este rango (Tabla 1). Es importante tener en consideración que con niveles elevados de $\mathrm{pH}$ la disponibilidad de ciertos nutrientes estará disminuida, afectando el desarrollo de los cultivos (Porta et al., 2003). $\mathrm{Al}$ respecto, Kang et al. (2011) establecieron que valores elevados de $\mathrm{pH}(>8)$ disminuyen la absorción de $\mathrm{P} \mathrm{y} \mathrm{Ca}{ }^{+2}$ por parte de los cultivos, y por el contrario aumenta la de $\mathrm{Mg}^{+2}$, debido a que se reduce la absorción de elementos competitivos con este catión. Por consiguiente, el pH en la zona de la raíz puede afectar directa o indirectamente el crecimiento, las características morfológicas y la absorción de agua y nutrientes.

$\mathrm{Si}$ bien la aplicación de los fertilizantes incide en el descenso del $\mathrm{pH}\left(\left(\mathrm{NO}_{3}\right)_{2} \mathrm{Ca} \mathrm{pH}\right.$ 5,87 y ácido fosfórico $\mathrm{pH}<1$ ), no se alcanzan valores considerados óptimos, por lo cual puede indicarse que el agregar ácido fosfórico, tal como se recomienda en la región con la finalidad de disminuir el pH (Balcaza, 2000), no provoca un efecto mejorador del $\mathrm{pH}$ que se mantenga en el tiempo y, por el contrario, genera un efecto negativo por la acumulación excesiva de $\mathrm{P}$ $\left(>100 \mathrm{mg} \mathrm{kg}^{-1}\right)$ conducente a desequilibrios nutricionales y contaminación (Cadahia, 2005). El aumento de $\mathrm{pH}$ también puede estar relacionado con las características de las enmiendas orgánicas incorporadas, ya que en la región de estudio se han observado en estos materiales valores de $\mathrm{pH}$ $>8$ (datos no mostrados).

Cationes intercambiables y sodicidad (PSI). En la Tabla 1 se presentan los valores de los cationes intercambiables (cmolc kg-1) y del PSI (\%) para todos los sitios evaluados. Respecto a los cationes intercambiables, se observa que los niveles de $\mathrm{K}^{+}$estuvieron en un rango entre 0,7- 4,2 cmolc $\mathrm{kg}^{-1} \mathrm{y}$ los de $\mathrm{Ca}^{+2}$ entre 12,3-34,1 cmolc $\mathrm{kg}^{-1}$, con promedios de 1,7 y 19,3 cmolc $\mathrm{kg}^{-1}\left(\mathrm{~K}^{+}\right.$y $\mathrm{Ca}^{+2}$, respectivamente). Se estima entonces que para la profundidad de muestreo $(0-15 \mathrm{~cm})$ y para los tipos de suelo de la región, las concentraciones

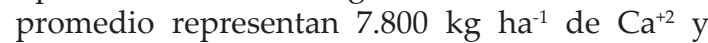
$1.300 \mathrm{~kg} \mathrm{ha}^{-1}$ de $\mathrm{K}^{+}$.

Rincón et al. (1993) establecieron que el cultivo de tomate y pimiento consumen en promedio 120 $\mathrm{kg} \mathrm{ha}^{-1} \mathrm{de} \mathrm{Ca}^{+2}$ y $460 \mathrm{~kg} \mathrm{ha}^{-1}$ de $\mathrm{K}_{2} \mathrm{O}$ (para producir $100 \mathrm{t}$ ), en tanto la lechuga posee requerimientos de $32 \mathrm{~kg} \mathrm{ha}^{-1}$ de $\mathrm{Ca}^{+2}$ y $196 \mathrm{~kg} \mathrm{ha}^{-1}$ de $\mathrm{K}_{2} \mathrm{O}$. Por lo tanto, con los valores aquí encontrados y los requerimientos de los cultivos, no sería necesario fertilizar. No obstante, se estableció que en promedio cada sitio aplicó $100 \mathrm{~kg} \mathrm{ha}^{-1}$ en forma de $\mathrm{CaO}$, aportado como $\left(\mathrm{NO}_{3}\right)_{2} \mathrm{Ca}$ y $230 \mathrm{~kg} \mathrm{ha}^{-1}$ de $\mathrm{K}^{+}$aportado como $\mathrm{KNO}_{3^{\prime}}$ conduciendo con estas prácticas de manejo a hiperfertilización, desequilibrios nutricionales, mayor incidencia de plagas y enfermedades y aumento de los costos (Altieri and Nicholls, 2003; Giuffré et al., 2004; Alconada et al., 2011). Coincidentemente, Flores et al. (2007) realizaron un análisis del balance de nutrientes en la región de estudio, y encontraron que se realizaban aplicaciones excesivas de fertilizantes (excesos de aplicaciones de hasta 600 $\mathrm{kg} \mathrm{ha}^{-1} \mathrm{de} \mathrm{N}$ ), producto de la fertilización en base a "recetas", sin considerar tipo de fertilizante, dosis, extracción de cada cultivo y riqueza del suelo. En otros sitios del mundo se han obtenido similares resultados; así, Hochmuth y Cordasco (2008) en un cultivo de tomate en Florida, experimentaron con diferentes dosis de $\mathrm{N}, \mathrm{P}$ y $\mathrm{K}$, y no encontraron respuesta favorable a dosis crecientes de fertilizantes, por el contrario, altas dosis de $\mathrm{N}$ produjeron un efecto negativo en el rendimiento. Sainju et al. (2003) indican también disminuciones de rendimientos con elevada fertilización. Consecuentemente la fertilización es un punto clave a tener en consideración en el manejo de los suelos bajo cubierta (Zhang et al., 2006; Alconada et al., 2011). No obstante, no sólo hay que considerar los valores absolutos, sino que también hay que analizar las relaciones entre 
Tabla 1. Valores de $\mathrm{CE}\left(\mathrm{dS} \mathrm{m}{ }^{-1}\right), \mathrm{pH}$, Cationes intercambiables $\left(\mathrm{K}^{+}, \mathrm{Na}^{+}, \mathrm{Ca}^{2+}, \mathrm{Mg}^{2+}\right)\left(\mathrm{cmolc} \mathrm{kg}^{-1}\right), \mathbf{P}(\mathrm{mg}$ $\mathrm{kg}^{-1}$ ) y MO (\%), para todos los sitios evaluados.

Table 1. Values of EC $\left(\mathrm{dS} \mathrm{m}{ }^{-1}\right), \mathrm{pH}$, interchangeable cations $\left(\mathrm{K}^{+}, \mathrm{Na}^{+}, \mathrm{Ca}^{2+}, \mathrm{Mg}^{2+}\right)\left(\mathrm{cmolc} \mathrm{kg}^{-1}\right), \mathrm{P}(\mathrm{mg}$ $\left.\mathrm{kg}^{-1}\right)$ and $\mathrm{OM}(\%)$ for all the evaluated sites.

\begin{tabular}{|c|c|c|c|c|c|c|c|c|c|}
\hline \multicolumn{10}{|c|}{ Cationes intercambiables } \\
\hline \multirow{2}{*}{\multicolumn{7}{|c|}{------- cmolc kg kn -------------------- }} & \multirow{2}{*}{$\begin{array}{c}\text { PSI } \\
\%\end{array}$} & \multirow{2}{*}{$\frac{P}{\mathrm{mg} \mathrm{kg}^{-1}}$} & \multirow[t]{2}{*}{ MO } \\
\hline & & & & & & & & & \\
\hline 1 & 1,2 & 7,1 & 1,8 & 12,3 & 10,3 & 1,0 & 3,7 & 227,0 & 3,5 \\
\hline 2 & 6,2 & 6,9 & 1,8 & 23,9 & 5,8 & 5,5 & 18,7 & 321,5 & 4,8 \\
\hline 3 & 9,2 & 6,9 & 1,3 & 21,4 & 6,2 & 5,5 & 21,2 & 205,0 & 4,1 \\
\hline 4 & 5,3 & 7,0 & 1,9 & 24,1 & 5,1 & 5,5 & 18,9 & 305,0 & 4,9 \\
\hline 5 & 7,1 & 6,8 & 1,7 & 23,7 & 6,4 & 5,5 & 18,6 & 338,0 & 4,7 \\
\hline 6 & 1,4 & 7,8 & $\mathrm{~s} / \mathrm{d}$ & $\mathrm{s} / \mathrm{d}$ & 6,6 & $\mathrm{~s} / \mathrm{d}$ & $\mathrm{s} / \mathrm{d}$ & 322,0 & 4,1 \\
\hline 7 & 2,5 & 7,7 & 1,8 & 17,1 & 7,0 & 4,9 & 23,4 & 413,0 & 3,4 \\
\hline 8 & 1,8 & 7,6 & 2,1 & 21,3 & 6,2 & 4,9 & 17,1 & 199,0 & 5,3 \\
\hline 9 & 1,9 & 7,9 & 1,7 & 18,4 & 7,0 & 4,9 & 18,5 & 378,0 & 3,1 \\
\hline 10 & 2,0 & 7,5 & 2,0 & 19,0 & 3,7 & 4,9 & 22,3 & 177,4 & $\mathrm{~s} / \mathrm{d}$ \\
\hline 11 & 2,0 & 7,5 & 1,4 & 17,2 & 4,2 & 4,9 & 22,3 & 181,4 & $\mathrm{~s} / \mathrm{d}$ \\
\hline 12 & 2,0 & 7,5 & 1,8 & 18,0 & 3,6 & 2,0 & 9,1 & 132,0 & $\mathrm{~s} / \mathrm{d}$ \\
\hline 13 & 2,0 & 7,5 & 2,0 & 19,4 & 4,6 & 2,2 & 10,0 & 535,7 & $\mathrm{~s} / \mathrm{d}$ \\
\hline 14 & 2,0 & 7,5 & 1,6 & 18,2 & 4,2 & 2,5 & 11,4 & 122,6 & $\mathrm{~s} / \mathrm{d}$ \\
\hline 15 & 2,1 & 7,5 & 1,8 & 19,4 & 5,2 & 0,5 & 2,3 & 196,8 & $\mathrm{~s} / \mathrm{d}$ \\
\hline 16 & 1,1 & 6,6 & 2,7 & 12,4 & 3,1 & 1,8 & 10,8 & 471,0 & 2,8 \\
\hline 17 & 0,7 & 7,4 & 2,0 & 16,6 & 11,8 & 0,7 & 3,7 & 163,8 & 3,2 \\
\hline 18 & 4,4 & 8,3 & 1,2 & 17,6 & 2,2 & 7,0 & 33,8 & 392,0 & 2,9 \\
\hline 19 & 3,4 & 7,8 & 1,3 & 21,1 & 1,0 & 5,4 & 28,6 & 145,0 & 2,3 \\
\hline 20 & 3,1 & 7,8 & 0,7 & 13,0 & 5,6 & 2,8 & 14,7 & 142,0 & 3,0 \\
\hline 21 & 1,6 & 8,5 & 1,0 & 18,5 & 1,8 & 4,9 & 21,3 & 286,0 & 2,9 \\
\hline 22 & 3,9 & 8,6 & 1,1 & 18,1 & 3,1 & 6,9 & 32,9 & 226,0 & 2,3 \\
\hline 23 & 2,2 & 8,6 & 0,8 & 16,3 & 3,1 & 5,5 & 28,5 & 185,0 & 2,5 \\
\hline 24 & 4,6 & 7,5 & $\mathrm{~s} / \mathrm{d}$ & 16,6 & 1,6 & 5,3 & $\mathrm{~s} / \mathrm{d}$ & 352,0 & 3,1 \\
\hline 25 & 5,9 & 7,9 & 1,5 & 19,1 & 3,0 & 9,1 & 28,6 & 277,0 & 3,1 \\
\hline 26 & 4,1 & 8,1 & 1,6 & 16,1 & 3,1 & 6,1 & 30,1 & 308,0 & 2,4 \\
\hline 27 & 0,8 & 8,3 & 0,7 & 21,1 & 2,5 & 4,8 & 23,3 & 131,0 & 2,1 \\
\hline 28 & 3,6 & 6,7 & 2,4 & 17,5 & 5,7 & 5,0 & 25,5 & 224,0 & 3,0 \\
\hline 29 & 0,7 & 7,8 & 0,9 & 22,7 & 3,8 & 2,6 & 14,1 & 100,0 & 3,6 \\
\hline 30 & 1,0 & 7,2 & 1,6 & 21,3 & 5,7 & 2,0 & 10,0 & 245,0 & 3,9 \\
\hline 31 & 2,4 & 6,2 & 1,8 & 21,3 & 7,6 & 1,9 & 8,9 & 248,0 & 4,7 \\
\hline 32 & 3,7 & 7,5 & 1,5 & 22,7 & 3,8 & 5,1 & 23,4 & 192,0 & 2,6 \\
\hline 33 & 4,4 & 7,8 & 1,8 & 34,1 & 6,6 & 6,0 & 26,9 & 206,0 & 3,0 \\
\hline 34 & 4,3 & 7,0 & 4,2 & 20,8 & 11,8 & 5,0 & 24,5 & 381,0 & 3,1 \\
\hline 35 & 4,7 & 7,2 & 2,8 & 20,8 & 5,7 & 5,6 & 28,1 & 234,0 & 3,0 \\
\hline 36 & 10,7 & 7,3 & 1,2 & 17,2 & 2,0 & 3,8 & 19,1 & 200,0 & 4,6 \\
\hline
\end{tabular}

los cationes, tal como se discute en los puntos siguientes.

Respecto al PSI y al $\mathrm{Na}^{+}$, si bien se observa en la Tabla 1 que el nivel mínimo de PSI fue de $2,3 \%$, correspondiéndose con una concentración de $\mathrm{Na}^{+}$de $0,5 \mathrm{cmolc}^{-1}$, el valor medio para los sitios evaluados fue de $19 \%$ PSI $\left(4 \mathrm{cmolc} \mathrm{kg}^{-1}\right.$ de $\mathrm{Na}^{+}$), con un valor máximo que alcanzó el $34 \%$
PSI (7 cmolc $\left.\mathrm{kg}^{-1} \mathrm{Na}^{+}\right)$. Por lo tanto, la sodicidad fue generalizada, destacándose que el $62 \%$ de los sitios presentaron valores mayores al 15\% de PSI. Los niveles elevados de $\mathrm{Na}^{+}$en el suelo se pueden deber principalmente a la calidad del agua de riego utilizada en la región, que es bicarbonatada sódica (con concentraciones de $\mathrm{Na}^{+}$elevadas, cercanas a 7,3 meq L $^{-1}$ ) (Alconada y Zembo, 2000) 
y en menor medida a las enmiendas orgánicas aplicadas (Barbazán et al., 2011), con valores de hasta $5000 \mathrm{mg} \mathrm{L}^{-1}$ de $\mathrm{Na}^{+}$en las camas de pollo utilizadas (datos no mostrados). Concentraciones altas de $\mathrm{Na}^{+}$en el suelo no sólo afectan su estructura (Cadahia, 2005), sino que además produce antagonismo nutricional y competencia entre nutrientes, influyendo en la absorción, transporte y distribución de elementos minerales en la planta, afectando su normal desarrollo (Mengel y Kirkby, 2000). Así, diversos autores señalan la importancia de mantener relaciones adecuada entre el $\mathrm{Na}^{+}$y el K+ (Bar-Tal et al., 2001; Supanjani, 2006) y el $\mathrm{Ca}^{+2}$ (Alemán et al., 2009; Turhan et al., 2009) a fin de evitar deficiencias inducidas.

En el presente estudio la relación $\mathrm{Na} / \mathrm{K}$ estuvo entre $0,6-7$, con un valor medio de 3 (correspondiendo a concentraciones medias de 4 y 1,7 cmolc $\mathrm{kg}^{-1}, \mathrm{Na}^{+}$y $\mathrm{K}^{+}$, respectivamente). Cuando los valores de $\mathrm{Na}^{+}$superaron la media $\left(4\right.$ cmolc $\left.\mathrm{kg}^{-1}\right)$, la relación $\mathrm{Na} / \mathrm{K}$ fue mayor a 2 . Es importante destacar que cuanto mayor es la relación entre ambos cationes mayor es el daño en los cultivos, debido a la toxicidad por $\mathrm{Na}^{+} \mathrm{y}$ a la menor absorción de $\mathrm{K}^{+}$. Diferentes estudios demuestran que a pesar de que la fertilización de $\mathrm{K}^{+}$disminuye la relación $\mathrm{Na} / \mathrm{K}$, no contrarresta los efectos negativos de la salinidad (Bar-Tal et al., 2001; Supanjani, 2006)

Respecto al $\mathrm{Ca}^{+2}$, en diversas fuentes se indica la importancia de mantener relaciones adecuadas con el $\mathrm{Na}^{+}$, a fin de evitar deficiencias inducidas por competencia de absorción catiónica (Alemán et al., 2009; Turhan et al., 2009). Cuando la concentración de $\mathrm{Na}^{+}$es elevada se presenta mayor incidencia de BER en los cultivos de fruto (De Pascale et al., 2003) y tip burn en los cultivos de hoja (Saure, 2001). En el presente estudio, si bien las relaciones entre ambos cationes fueron bajas (menor a 0,4 ), por el elevado contenido de $\mathrm{Ca}^{+2}$ (concentraciones medias 19,5 $\mathrm{cmolc} \mathrm{kg}^{-1}$ ), los cultivos han presentado deficiencias inducidas, debido a que el $\mathrm{Ca}^{+2}$ puede interactuar con otros cationes (como por ejemplo $\mathrm{Mg}^{+2}$ ) y disminuir su disponibilidad (Mengel y Kirkby, 2000).

No sólo es importante las interacciones de los diferentes cationes del suelo con el $\mathrm{Na}^{+}$, sino que también hay que mantener ajustada la proporción entre los mismos (Cadahia, 2005). El $\mathrm{Mg}^{+2}$ se absorbe rápidamente por la raíz y compite por los sitios de absorción con el $\mathrm{Ca}^{+2}$ (Marschner, 1995). Se establece que si la relación $\mathrm{Ca} / \mathrm{Mg}$ es $<1$ habrá deficiencia de $\mathrm{Ca}^{+2}$, y si la misma es $>10$ la deficiencia será de $\mathrm{Mg}^{+2}$. En el análisis realizado se determinó que en promedio, la relación entre ambos cationes fue próxima a 5 , con mínimos que se encontraron en 1,2 y máximos que llegaron a
21. Por lo tanto, excepto dos sitios en los cuales se pudo llegar a presentar deficiencia de $\mathrm{Mg}^{+2}$, para el resto la relación entre ambos cationes fue adecuada.

La deficiencia de $\mathrm{Mg}^{+2}$ en los cultivos se puede deber no sólo a la baja disponibilidad en el suelo (baja fertilización, inmovilización), sino también a la competencia con otros cationes (principalmente con $\mathrm{NH}_{4}^{+}, \mathrm{Ca}^{+2} \mathrm{y} \mathrm{K}^{+}$). En general el $\mathrm{Mg}^{+2}$ por sus propiedades químicas, queda menos retenido en el complejo de intercambio, y consecuentemente hay mayor concentración en la solución del suelo, por lo tanto al tener mayor movilidad y concentración respecto a los otros cationes, se lixivia con facilidad, favoreciéndose así deficiencias inducidas (Mengel y Kirkby, 2000; Gransee and Führs, 2013). Sin embargo, se destaca que en valores absolutos las concentraciones de $\mathrm{Mg}^{+2}$, tanto en los sitios de estudio (concentraciones medias $4,38 \mathrm{cmolc} \mathrm{kg}^{-1}$ ) como en los del suelo natural, resultan suficientes para una adecuada nutrición magnésica (Hurtado et al., 2006).

Es importante mencionar que existe además entre el $\mathrm{Mg}^{+2}$ y el $\mathrm{K}^{+}$un fuerte efecto antagónico (Gransee and Führs, 2013). La relación $\mathrm{Mg} / \mathrm{K}$ se debe encontrar entre 2,5-5; si es menor habrá deficiencia de $\mathrm{Mg}^{+2}$ (Havlin et al., 1999). Estrada (2001) estableció como ideal un valor de $3(\mathrm{Mg} / \mathrm{K})$. El valor promedio fue de 3, destacándose que aproximadamente el $50 \%$ de los sitios presentaron valores menores. Por consiguiente, según el criterio establecido por Halvin et al. (1999), estos sitios podrían presentar deficiencias inducidas de $\mathrm{Mg}^{+2}$ por las concentraciones de $\mathrm{K}^{+}$.

Otra relación importante es la que se produce entre el $\mathrm{Ca}^{+2}$ y el $\mathrm{K}^{+}$. Mengel y Kirkby (2000) señalan que si bien el $\mathrm{Ca}^{+2}$ respecto al $\mathrm{K}^{+}$en solución edáfica es 10 veces mayor, la tasa de absorción de $\mathrm{Ca}^{+2}$ es menor que la de $\mathrm{K}^{+}$. Esto se debe a que el $\mathrm{Ca}^{+2}$ se absorbe por las células más jóvenes de la raíz, mientras que el $\mathrm{K}^{+}$por todas las células. Por consiguiente, niveles elevados de $\mathrm{K}^{+}$pueden afectar la absorción de $\mathrm{Ca}^{+2}$, por lo que relaciones $\mathrm{K} / \mathrm{Ca}$ altas aumentan la incidencia de BER (Taylor et al., 2004, Rubio et al., 2009). En el análisis de los sitios evaluados, se observó que esta relación en promedio estuvo en un valor de 13 , siendo algo elevada para los valores ideales $(\mathrm{Ca} / \mathrm{K}=6)$ establecidos por Estrada (2001); asimismo este autor manifiesta que con valores superiores a 25 se producirá deficiencia de $\mathrm{K}^{+}$, lo ocurrido sólo en dos de los sitios.

Fósforo En la Tabla 1 se presentan los valores de P asimilable (BK I). En la misma se observan concentraciones de entre 100 y $535 \mathrm{mg} \mathrm{kg}^{-1}$, siendo el valor promedio de $253 \mathrm{mg} \mathrm{kg}^{-1}$. Se destaca 
que los suelos de la región en su condición natural se caracterizan por tener un nivel bajo de $\mathrm{P}$ asimilable ( $<\mathrm{a} 10 \mathrm{mg} \mathrm{kg}^{-1}$ ) (Hurtado et al., 2006). Los valores encontrados fueron similares a los reportados $\left(300 \mathrm{mg} \mathrm{kg}^{-1}\right)$ en la región de estudio (Giuffré el at., 2004). No obstante, y aún con estas concentraciones elevadas, es común que se incorpore $\mathrm{P}$ en la fertilización de base, o por medio de la incorporación de ácido fosfórico durante el desarrollo del cultivo. Sin embargo, con concentraciones tan elevadas en el suelo no se encuentra respuesta a la fertilización (Balemi and Negisho, 2012) y, por el contrario, se conduce a situaciones de hiperfertilización, tal como las reportadas por Giuffré et al. (2004) y Alconada et al. (2006). La disponibilidad de P no sólo depende de la fertilización química, sino que también de la respuesta o de la interacción con el $\mathrm{Ca}^{+2}, \mathrm{Mg}^{+2}$, $\mathrm{Al}^{+3}, \mathrm{Fe}^{+3} \mathrm{y} \mathrm{Mn}^{+2}$ (Carrijo and Hochmuth, 2000). Así, en situaciones de $\mathrm{pH}$ elevados, tal como las manifestadas en este estudio, el $\mathrm{P}$ forma compuestos insolubles con el $\mathrm{Ca}^{+2}$ y el $\mathrm{Mg}^{+2}$ (Balemi and Negisho, 2012), conduciendo a deficiencias inducidas.

Otra fuente de $\mathrm{P}$ al suelo, es la incorporación de camas de pollo o gallinaza, que se realiza en la región una o dos veces por año para mejorar el contenido de materia orgánica del suelo y así mejorar su estructura. Sin embargo, no se consideran las características físico-químicas de estos materiales y el efecto que tienen en el suelo. Estos materiales son muy variables en su composición, pero en general en la región de estudio aportan en promedio $8,7 \mathrm{~g}^{\mathrm{de}} \mathrm{P} \mathrm{kg}{ }^{-1}$ (Alconada, 2004), aunque se han detectado en otros sitios concentraciones más elevadas, de 25,1 y $13,1 \mathrm{~g} \mathrm{~kg}^{-1} \mathrm{de} \mathrm{P}$, para cama de gallina y gallinaza, respectivamente (Barbazán et al., 2011).

Materia orgánica (MO) En la Tabla 1 se presentan los valores de Materia Orgánica (\%) para todos los sitios evaluados, alcanzando un nivel promedio de 3,4\%, con mínimos de 2,1\%. Los niveles de MO fueron menores a los encontrados en los suelos de la región en su condición natural $(>4 \%$ ) (Hurtado et al., 2006).

Cabe destacar que todos los sitios regularmente efectúan un agregado continuo de cama de gallina o de gallinaza. Sin embargo, esto no conduce a aumentos significativos de materia orgánica, ya que los materiales orgánicos formadores de humus son los de origen vegetal (Labrador Moreno, 1996). Así, se manifiestan como principales inconvenientes en el uso de este tipo de materiales: la falta de conocimiento técnico, de análisis y seguimiento en la aplicación, la dosificación inadecuada, el desconocimiento acerca del tiempo de degradación y del impacto que estas prácticas tienen en el cultivo. Diferentes autores destacan que al aplicar estos abonos en altas dosis se visualizan efectos adversos, como aumento de la salinización del suelo (Sasal et al., 2000; Salazar Sosa et al., 2004; Pérez et al., 2008).

\section{CONCLUSIONES}

Se concluyó que los sitios analizados presentan serios problemas de manejo de los suelos en invernaderos. Se encontraron elevados valores de salinidad, con promedios de 3,8 dS $\mathrm{m}^{-1}$ y extremos de $10,7 \mathrm{dS} \mathrm{m}^{-1}$; la sodificación fue también elevada (valores medios $18 \%$ de PSI), y el $\mathrm{pH}$ aumentó de 5,5 en su condición natural a valores de 7,5. Se destaca que las aplicaciones en exceso de fertilizantes y enmiendas orgánicas sin un ajuste a los tipos de suelo de la región y a las necesidades de los cultivos condujeron a situaciones de hiperfertilización (concentraciones de $\mathrm{P}$ extremas de $535 \mathrm{mg} \mathrm{kg}^{-1}$ ) lo que genera riesgos de deficiencias inducidas.

Si bien este trabajo representó una porción del universo de productores de la región, se visualiza que los suelos del Gran La Plata sufren una situación de degradación intensa con posibles contaminaciones. Por consiguiente, es muy importante ajustar la forma de manejo, para no comprometer la producción de hortalizas periurbana y la sustentabilidad del sistema productivo.

\section{LITERATURA CITADA}

Alconada, M. 2004. Desinfección del suelo con vapor. Efectos sobre la nutrición de los cultivos. p. 124. Proyecto Tierra Sana MP/ARG/00/033. INTA, Ciudad Autónoma de Buenos Aires, Argentina.

Alconada, M., M. Cuellas, P. Poncetta, S. Barragán, E. Inda, y A. Mitidieri. 2011. Fertirrigación en un cultivo de tomate protegido: I-Nutrición nitrogenada. Efectos en el suelo y en la producción. Revista Horticultura Argentina 30(72):5-13.

Alconada, M., P. Poncetta, M. Cuellas, S. Barragán, E. Inda, y A. Mitidieri. 2006. La fertirrigación en cultivo de tomate protegido (Lycopersicum esculentum): consecuencias ambientales, productivas y económicas. En XX Congreso Argentino de la Ciencia del Suelo, y I Reunión de Suelos de la Región Andina. 20-22 de Septiembre. Asociación Argentina de Ciencia del Suelo (AACS), Salta, Argentina. 
Alconada, M., y J. Zembo. 2000. Influencia cualitativa del riego con aguas subterráneas en suelos con producciones intensivas a campo y en invernáculo. En Actas 1er Joint World Congress on Groundwater. 31 de Julio-4 de Agosto. Northeastern Brazil Groundwater Project (PROASNE). Associação Brasileira de Aguas Subterrâneas (ABAS), Fortaleza, Brasil.

Alemán, F., C. Nieves, M. Martínez, and F. Rubio. 2009. Potassium/sodium steady-state homeostasis in Thellungiela halophila and Arabidopsis thaliana under long-term salinity conditions. Plant Science 176:768-774.

Altieri, M., and C. Nicholls. 2003. Soil fertility management and insect pests: harmonizing soil and plant health in agroecosystems. Soil \& Tillage Research 72:203-211.

Auge, M., R. Hirata, y F. López Vera. 2004. Vulnerabilidad a la contaminación por nitratos del acuífero puelche en La Plata, Argentina. 187 p. Informes del Centro de Estudios de América Latina (CEAL). Disponible en http://tierra. rediris.es/hidrored/ebooks/miguel/Nitratos.pdf (Consulta 10 abril 2016).

Balcaza, L. 2000. Utilización de ácidos en fertirriego. Boletín Hortícola. F.C.A, UNLP- INTA, Gran Buenos Aires 8(26):13-15.

Balemi, J., and K. Negisho. 2012. Management of soil phosphorus and plant adaptation mechanisms to phosphorus stress for sustainable crop production: a review. Journal of Soil Science and Plant Nutrition 12(3):547-561.

Barbazán, M., A. del Pino, C. Moltini, J. Hernández, y J. Rodríguez. 2011. Caracterización de materiales orgánicos aplicados en sistemas agrícolas intensivos de Uruguay. Agrociencia Uruguay 15(1):82-92.

Barsky, A. 2005. El periurbano productivo, un espacio en constante transformación. Introducción al estado del debate, con referencias al caso de Buenos Aires. Revista Electrónica de Geografía y Ciencias Sociales 9(194):36.

Bar-Tal, A., B. Aloni, L. Karni, J. Oserovitz, A. Hazan, M. Itach, et al. 2001. Nitrogen nutrition of greenhouse pepper. I Effects of nitrogen concentration and $\mathrm{NO}_{3}: \mathrm{NH}_{4}$ ratio on yield, fruit shape, and the incidence of Blossom-end rot in relation to plant mineral composition. HortScience 36(7):1244-1251.

Cadahia, L.C. 2005. Fertirrigación: Cultivos hortícolas y ornamentales. 681 p. 3ra ed. Mundi Prensa, Madrid, España.

Carrijo, O., and G. Hochmuth. 2000. Tomato responses to preplant-incorporated or fertigated phosphorus on soils varying in Mehlich-1 Extractable Phosphorus. HortScience 35(1):67-72.
Cuellas, M. 2015. Control dela salinización del suelo mediante sistemas de drenes en producciones intensivas de cultivos bajo cubierta. 162 p. M. Sc. Tesis. Facultad de Agronomía, Universidad de Buenos Aires. Buenos Aires, Argentina.

De Pascale, S., C. Ruggiero, G. Barbieri, and A. Maggio. 2003. Physiological responses of pepper to salinity and drought. Journal of the America Society for Horticultural Science 128(1):48-54.

Del Amor, F.M., and L.F.M. Marcelis. 2006. Differential effect of transpiration and $\mathrm{Ca}$ supply on growth and $\mathrm{Ca}$ concentration of tomato plants. Scientia Horticulturae 111(1):1723.

Estrada, E. 2001. Disponibilidad de calcio, magnesio y azufre, su análisis en suelos y plantas y su interpretación. p. 85-104. En Silva, M. (ed.) Los elementos secundarios ( $\mathrm{Ca}, \mathrm{Mg}, \mathrm{S})$ y el silicio en la agricultura. Sociedad Colombiana de la Ciencia del Suelo, Comité Regional de Cundinamarca y Boyacá, Bogotá, Colombia.

Flores, C., S. Sarandón, y N. Gragoloff. 2007. Balance simplificado de nutrientes como indicador de la sustentabilidad en sistemas hortícolas familiares del partido de La Plata, Buenos Aires, Argentina. II Congresso Brasileiro de Agroecológica. Revista Brasileira Agroecológica 2(1):1065-1068.

Giuffré, L., M. Alconada, C. Pascale, and S. Ratto. 2004. Environmental impact of phosphorus overfertilization in tomato greenhouse production. Journal Applied Horticulture 6(1):58-61.

Gransee, A., and H. Führs. 2013. Magnesium mobility in soils as a challenge for soil and plant analysis, magnesium fertilization and root uptake under adverse growth conditions. Plant Soil 368:5-21.

Havlin, J.L., J.S. Beaton, S.L. Tisdale, and W.L. Nelson. 1999. Soil Fertility and Fertilizers. 499 p. 6th ed. Prentice Hall, Upper Saddle River, New Jersey, USA.

Ho, L.C., and P. White. 2005. A cellular hypothesis for the induction of blossom-end rot in tomato fruit. Annals of Botany 95:571-581.

Hochmuth, G., and K. Cordasco. 2008. A summary of N, P, and K Research with Tomato in Florida. Series, Horticultural Sciences Department, Cooperative Extension Service, Institute of Food and Agricultural Sciences, University of Florida. HS 759. Available at http://edis.ifas.ufl. edu (Consulta 5 marzo 2016).

Hurtado, M, J. Giménez, y M. Cabral. 2006. Análisis ambiental del partido de La Plata: Aportes al ordenamiento territorial. 134 p. Consejo Federal de Inversiones, La Plata, Buenos Aires, Argentina. 
INTA. 2011a. Diagnóstico de la cadena de valor florícola en el área del centro regional Buenos Aires norte. Instituto Nacional de Tecnología Agropecuaria (INTA) PTR 2012- 2015. INTA, Buenos Aires, Argentina.

INTA, 2011b. Diagnóstico territorio Periurbano Sur. PTR 2012- 2015. EEA AMBA. Centro regional Buenos Aires norte. Instituto Nacional de Tecnología Agropecuaria (INTA), Buenos Aires, Argentina.

Johnstone, P.R., T.K. Hartz, M.D. Cahn, and M.R. Johnstone. 2005. Lettuce response to phosphorus fertilization in high phosphorus soils. HortScience 40(5):1499-1503.

Kang, Y., J. Park, S. Kim, N. Kang, K. Park, S. Lee, et al. 2011. Effects of root zone $\mathrm{pH}$ and nutrient concentration on the growth and nutrient uptake of tomato seedlings. Journal of Plant Nutrition 34:640-652.

Kurunc, A., A. Unlukara, and B. Cemek. 2014. Salinity and drought affect yield response of bell pepper similarly. Acta Agriculturae Scandinavica, section B. Soil \& Plant Science 61(6):514:522.

Labrador Moreno, J. 1996. La materia orgánica en los agroecosistemas. 174 p. Mundi Prensa, Madrid, España.

López Camelo, A. 2011. Relevamiento satelital de los invernaderos en el cinturón hortícolas de la plata. XXXIV Congreso Argentino de Horticultura. 27-30 de Septiembre. Asociación Argentina de Horticultura (ASAHO), Buenos Aires, Argentina.

Maggio A., G. Raimondi, A. Martino, and S. De Pascale. 2007. Salt stress response in tomato beyond the salinity tolerance threshold. Environmental and Experimental Botany 59:276-282.

Marschner, H. 1995. Mineral nutrition of higher plants. 889 p. 2da ed. Academic Press, San Diego, California, USA.

Mengel, K., y E.A. Kirkby. 2000. Principios de nutrición vegetal. 535 p. 4ta ed. Instituto Internacional de la Potasa, Basilea, Suiza.

Nuez, F., G. Ortega, y J. Costa. 2003. El cultivo de pimientos, chiles y ajíes. 607 p. Mundi Prensa, Madrid, España.

Page, A., A.H. Miller, and D.R. Keeney. 1982. Methods of Soils Analysis. Soil Sci. Soc. America, Madison, Wisconsin, USA.

Pérez, A., C. Céspedes, y P. Muñoz. 2008. Caracterización física-química y biológica de enmiendas orgánicas aplicadas en la producción de cultivos en República Dominicana. R. C. Suelo Nutr. Vegetal 8(4):1029.
Polack, L. A. 2013. Tecnología apropiada para la sustentabilidad de sistemas hortíflorícolas con énfasis en cultivos protegidos. (PNHFA 1106082). PTR 2013-2019. Instituto Nacional de Tecnología Agropecuaria (INTA), Buenos Aires, Argentina.

Poncetta, P., M. Alconada, y R. Lavado. 2006. Producción de tomate protegido en suelos decapitados con diferentes planes de fertirrigación. En XX Congreso Argentino de la Ciencia del Suelo y I Reunión de Suelos de la Región Andina. 20-22 de Septiembre. Asociación Argentina de Ciencia del Suelo (AACS), Salta, Argentina.

Porta, J., M. López Acevedo, y C. Roquero. 2003. Edafología para la agricultura y el medio ambiente. 930 p. 3ra ed. Mundi Prensa, Madrid, España.

Rincón, L., J. Saez., E. Balsalobre, y M. Pellicer. 1993. Nutrición del pimiento grueso de invernadero. Hortofruticultura 5:37-41.

Rubio, J.S., F. García-Sánchez, F. Rubio, and V. Martínez. 2009. Yield, blossom-end rot incidence, and fruit quality in pepper plants under moderate salinity are affected by $\mathrm{K}^{+}$ and $\mathrm{Ca}^{2+}$ fertilization. Scientia Horticulturae 119:79-87

Sainju, U M., R. Dris, and B. Singh. 2003. Mineral nutrition of tomato. International Journal of Food, Agriculture and Environment 3(1):176183.

Salazar Sosa, E., C. Vázquez Vázquez, J. Leos Rodríguez, M. Fortis Hernández; J. Montemayor Trejo, R. Figueroa Viramontes, et al.2004. Mineralización del estiércol bovino y su impacto en la calidad del suelo y la producción de tomate (Lycopersicum esculentum Mill.) bajo riego sub-superficial. Phyton 73:259-273.

Sasal, C., A. Andriulo, J. Ullé, F. Abrego, y M. Bueno. 2000. Efecto de diferentes enmiendas sobre algunas propiedades edáficas en sistemas de producción hortícola del centro norte de la Región Pampeana. Ciencia del Suelo 18(2):95-104.

Saure, M.C. 2001. Review. Blossom-end rot of tomato (Lycopersicon esculentum Mill.)- a calcium- or a stress-related disorder?. Scientia Horticulturae 90(3-4):93-208.

Supanjani, K.D. 2006. Hot pepper response to interactive effects of salinity and boron. Plant Soil and Environment 52(5):227-233.

Szczesny, A., E. Adlercreutz, D. Huarte, A. López Camelo, E. Manzo, y L. Viglianchino. 2014. Producción hortícola bajo cubierta 150 p. Instituto Nacional de Tecnología Agropecuaria (INTA), Ciudad Autónoma de Buenos Aires, Argentina. 
Taylor, M.D., S.J. Locassio, and M.R. Alligood. 2004. Blossom-end rot incidence of tomato as affected by irrigation quantity, calcium source, and reduced potassium. HortScience 39(5):1110-1115.

Turhan, A., V. Seniz, and H. Kuscu. 2009. Genotypic variation in the response of tomato to salinity. African Journal of Biotechnology 8(6):1062-1068.

Ünlükara, A., B. Cemek, S. Karaman, and S. Erşahin. 2010. Response of lettuce (Lactuca sativa var. crispa) to salinity of irrigation water. New Zealand Journal of Crop and Horticultural Science 36(4):265-273.

Zhang, Y., Y. Jiang, and W. Liang. 2006. Accumulation of soil soluble salt in vegetable greenhouses under heavy application of fertilizers. Agricultural Journal 1(3):123-127. 